\title{
Research on Emission Reduction Strategy of Manufacturer Leading Low-Carbon Supply Chain Under Fair Concern
}

\author{
Hongqi Wang ${ }^{1}$, Xingbo Qiu ${ }^{1 *}$ \\ ${ }^{1}$ School of Management, Xinhua College of Sun Yat-Sen University, Guangzhou, Guangdong, 510520, China \\ *Corresponding author. Email: wanghongqi1001@163.com
}

\begin{abstract}
The emission reduction strategy of manufacturer-led structure is studied, and the differences of emission reduction level, retail price and profit are analysed under fair neutrality and fair concern. Research shows that the emission reduction cost factor changes in the opposite direction with emission reduction level, retail price and profit. There is a conflict between the manufacturer's fair concern behaviour and the dominant structure. Fair concern is not conducive to the improvement of emission reduction and profit level, but conducive to the increase of retail price. The coordination of supply chain can be realized through the two-part pricing contract model. Finally, the effects of equity concern factor and emission reduction cost factor on emission reduction level, retail price and profit level are verified by numerical simulation.
\end{abstract}

Keywords: Low carbon supply chain, manufacturer-led, fair concern, emission reduction strategy

\section{INTRODUCTION}

With global warming, consumers' demand for low-carbon products is growing, which urges all enterprises in the production sector to take various emission reduction measures, but the overall effect is modest. In practice, it is generally believed that manufacturers bear the main responsibility for emission reduction, while retailers help to share the cost of emission reduction. The research shows that the cost sharing process of emission reduction will be influenced by members' fair concern. Another part of scholars introduced equity concern into low-carbon supply chain studies. Cui etal. [1] introduced equity concern into the supply chain earlier, considering the supply chain coordination problem when members have equity concern. Du etal. [2] took Nash negotiation solution as a fair neutral point, and studied the newsboy problem that many members were concerned about. Subsequent scholars extended equity concerns to low-carbon supply chains. For example, Li etal. [3] considered the coordination of low-carbon supply chains in which members have equity concerns on the premise of tradable carbon emissions. Liu etal. [4] compared and analysed the pricing strategy and coordination strategy of low-carbon supply chain under the consideration of fairness, neutrality and fairness. Under the manufacturer's equity concern, Shi etal. [5] analysed the impact of equity concern coefficient and low carbon coefficient on carbon emissions, member pricing strategies and supply chain efficiency. Zhou etal. [6] analysed the influence of the coefficient of equity concern, the efficiency of product greenization and market share on the decision-making and profit of each member, taking into account the retailer's equity concern. Zhou etal. [7] also considered the optimal coordination strategy of advertising cooperation -- emission reduction cost sharing under the concern of manufactuer-led structure and retailers' equity. Zhou etal. [8] analysed the impact of lowcarbon supply chain optimal pricing strategy and product greenness decision under the concern of retailers' leading channels and manufacturers' equity.

From the above studies, it can be found that the core issues of low-carbon supply chain are the allocation of emission reduction costs and the coordination of supply chain. Some scholars solve this problem from the coordination method of supply chain itself, while others discuss the coordination of supply chain from the perspective of members' equity concerns or channel power structure. However, there are few literatures that study from the perspective of fairness concern and manufacturer-led structure at the same time. Therefore, this paper studies the optimal decision of manufacturer-led structure and the behaviour with fairness concern at the same time.

\section{BASIC MODEL AND HYPOTHESIS}

The paper considers a secondary supply chain composed of a manufacturer and a retailer. Considering the low carbon preference of consumers, market demand is a linear function of product price and emission reduction. On this basis, the strategies of emission reduction under the consideration of fairness neutrality and fairness were studied. In order to distinguish the profit functions of manufacturers, retailers and the whole supply chain, variables $m, r$ and $s c$ were used as subscripts. The variables under equity concern take "*" as the superscript, and the equilibrium value of the centralized decision under equity neutrality takes $c$ as the subscript. The parameters involved in the paper are as follows: 
Table 1 Notations

\begin{tabular}{|l|l|}
\hline Parameter & \multicolumn{1}{c|}{ Definition } \\
\hline$a$ & market size \\
\hline$b$ & consumers sensitivity to price \\
\hline$\varepsilon$ & low carbon preference coefficient \\
\hline$e$ & emission levels \\
\hline$w$ & wholesale price \\
\hline$c$ & manufacturer's cost \\
\hline$\rho$ & retailer's profit per item \\
\hline$k$ & emission reduction cost factor \\
\hline
\end{tabular}

Assumption 1. The retailer's sales process is passive, without considering the sales effort, and the cost is zero, so it can be known that $p=\rho+w$, and $p>w>c$. Therefore, $a>b p>b c$, combined with the manufacturer and retail profits discussed in the paper, is a concave function of the decision-making variable, so we set $2 b k \geq \varepsilon^{2}$.

Assumption 2. Referring to the study of Zhou [8], we can assume that the cost of emission reduction is $k e^{2} / 2$. For ease of expression, Let's say $N=a-b c, M_{1}=2 b k-\varepsilon^{2}$,

$$
M_{2}=4 b k-\varepsilon^{2}, L=2 b k[2(1+\beta)+\beta \gamma]-(1+\beta) \varepsilon^{2} .
$$

Thus, the demand function and profit function can be obtained:

$$
\begin{array}{r}
Q=a-b(w+\rho)+\varepsilon e \\
\pi_{m}=(w-c)[a-b(w+\rho)+\varepsilon e]-\frac{1}{2} k e^{2} \\
\pi_{r}=\rho[a-b(w+\rho)+\varepsilon e] \\
\pi_{s c}=(w+\rho-c)[a-b(w+\rho)+\varepsilon e]-\frac{1}{2} k e^{2}
\end{array}
$$

The centralized decision-making process is as follows. According to equation (4), the first and second partial derivatives of $\pi_{s c}$ with respect to $e, p$ can be obtained The Hesse matrix $H\left(p_{c}, e_{c}\right)=\left[\begin{array}{cc}-2 b & \varepsilon \\ \varepsilon & -k\end{array}\right]$, According to the matrix, the first order principal sub formula $-2 b<0$ and the second order principal sub formula $2 b k-\varepsilon^{2}>0$, we can determine that $H\left(p_{c}, e_{c}\right)$ is a negative definite matrix.

Therefore, there are optimal solutions for $p_{c}$ and $e_{c}$, by solving the equation group : $\left\{\begin{array}{l}a+\varepsilon e_{c}+b c-2 b p_{c}=0 \\ \varepsilon\left(p_{c}-c\right)-k e_{c}=0\end{array}\right.$

we can be obtained: $e_{c}=\varepsilon N / M_{1}, p_{c}=c+k N / M_{1}$, $\pi_{c s c}=k N^{2} / 2 M_{1}$.

\section{OPTIMAL DECISION UNDER MANUFACTURER-LED STRUCTURE}

\subsection{Optimal decision under fairness neutrality}

In the Stackelber game structure dominated by manufacturers, manufacturers first determine the wholesale price and emission reduction level, and retailers determine the profit per unit product according to the wholesale price and emission reduction level. The inverse order method is used to solve the problem. The first derivative with respect to $\rho$ is obtained from equation (3).

Set it equal to zero to get: $\rho=(a+\varepsilon e-b w) / 2 b$.

Substitute $\rho$ into equation (2) to obtain the manufacturer's profit function, and take the first, second and partial derivatives with respect to $w, e$ with respect to $\pi_{m}$.The Hesse matrix of $\pi_{m}$ can be obtained as follows: $H(w, e)=\left[\begin{array}{cc}-b & \varepsilon / 2 \\ \varepsilon / 2 & -k\end{array}\right]$, According to the matrix, the first order principal sub formula $-b<0$ and the second order principal sub formula $4 b k-\varepsilon^{2}>0$, we can determine that $H(w, e)$ is a negative definite matrix. Therefore, there are optimal solutions for $w$ and $e$,by solving the equation group : $\left\{\begin{array}{l}-b w+(a+\varepsilon e+b c) / 2=0 \\ -k e+\varepsilon(w-c) / 2=0\end{array}\right.$

we can be obtained: $e=\varepsilon N / M_{2}, w=c+2 k N / M_{2}$, $\rho=k N / M_{2}$.

Substitute $w, e$ and $\rho$ into equation (2) - equation (4) to get: $\quad \pi_{m}=k N^{2} / 2 M_{2} \quad, \quad \pi_{r}=b k^{2} N^{2} / M_{2}{ }^{2}$, $\pi_{s c}=k N^{2}\left(6 b k-\varepsilon^{2}\right) / 2 M_{2}^{2}$

\subsection{Optimal decision under equity concern}

According to the equity concern theory, when considering their own profits, supply chain members will also compare the profits of other members. $\gamma \pi_{r}$ is the reference point of the manufacturer's fair profit. When $0<\gamma \pi_{r}<\pi_{m}$, the manufacturer's profit is higher than the reference point of fair profit, resulting in positive unfairness. On the contrary, it will produce negative unfairness. Research has shown that members are more concerned about inequity that is detrimental to them. Here, only the case where the manufacturer's profit is less than the reference point of fair profit is considered. Referring to the study of Cui etal. [1], the manufacturer's utility function can be expressed as:

$u_{m}=\pi_{m}-\beta\left(\gamma \pi_{r}-\pi_{m}\right), \quad \beta(0<\beta<1)$ is the manufacturer's equity concern factor, and the utility functions of the manufacturer and the retailer are: 


$$
\begin{gathered}
u_{r}=\pi_{r}=\rho(a-b \rho-b w+\varepsilon e) \\
u_{m}=(1+\beta)\left[(w-c)(a-b \rho-b w+\varepsilon e)-\frac{1}{2} k e^{2}\right] \\
-\beta \gamma \rho(a-b \rho-b w+\varepsilon e)
\end{gathered}
$$

The problem solving process under the concern of equity is similar to 3.1. According to equations (5) and (6), the following can be obtained: $w^{*}=c+2 k(1+\beta+\beta \gamma) N / L$

$e^{*}=\varepsilon(1+\beta) N / L, \rho^{*}=k(1+\beta) N / L$.

Substitute $w^{*}, e^{*}$ and $\rho^{*}$ into equation (2) -(4) to get:

$$
\begin{array}{r}
\pi_{m}^{*}=k(1+\beta) N^{2}(2 b k \beta \gamma+L) / 2 L^{2}(7) \\
\pi_{r}^{*}=b k^{2}(1+\beta)^{2} N^{2} / L^{2}(8) \\
\pi_{s c}^{*}=k(1+\beta) N^{2}[2 b k(1+\beta+\beta \gamma)+L] / 2 L^{2}
\end{array}
$$

And then you get the manufacturer's utility:

$u_{m}^{*}=k(1+\beta)^{2} N^{2} / 2 L$, The constraint condition can be obtained according to the known condition $\gamma \pi_{r}>\pi_{m}>0$ : $\gamma>2-\varepsilon^{2} / 2 b k, \beta<\left(2 b k \gamma-M_{2}\right) /\left(2 b k \gamma+M_{2}\right)$.

In order to further study the conditions for manufacturers to maximize utility, set $\partial u_{m}^{*} / \partial \beta=0$, We know that when $\beta=\left(2 b k \gamma-M_{2}\right) /\left(2 b k \gamma+M_{2}\right)$, the manufacturer has the most utility.

\subsection{Comparison and coordination analysis}

Proposition 1: fair concern is not conducive to the level of emission reduction and the improvement of supply chain members' profits.

Proving Course: according to the previous calculation results, if we calculate $e^{*}-e, \pi_{r}^{*}-\pi_{r}$ and $\pi_{m}^{*}-\pi_{m}$, we can get:

$$
\begin{aligned}
& e^{*}-e=-2 b k \beta \gamma \varepsilon N / M_{2} L<0 \\
& \pi_{r}^{*}-\pi_{r}=-2 b^{2} k^{3} N^{2} \beta \gamma\left[(1+\beta) M_{2}+L\right] / M_{2}{ }^{2} L^{2}<0 \\
& \pi_{m}^{*}-\pi_{m}=-4 b^{2} k^{2} \beta^{2} \gamma^{2} / M_{3}{ }^{2} L_{1}<0
\end{aligned}
$$

Proposition 2: fair care is conducive to the increase of retail prices.

Proving Course: according to the previous calculation results, if we calculate $p^{*}-p$, we can get:

$$
p_{1}^{*}-p_{1}=2 k \beta \gamma N\left(b k-\varepsilon^{2}\right) / M_{2} L>0
$$

Proposition 3: Supply chain coordination can be realized in two pricing contracts. When $0 \leq F<b k^{2} N^{2} / M_{1}^{2}$ and $w_{c}=c$.

Proving Course: In the two-part pricing contract model, the retailer pays a one-time fixed fee $F$ to the manufacturer as the cost of emission reduction. At this time, the profit of the manufacturer and the retailer is:

$$
\pi_{m c}=(w-c)[a-b(w+\rho)+\varepsilon e]-k e^{2} / 2+F(10)
$$

$$
\pi_{r c}=\rho[a-b(w+\rho)+\varepsilon e]-F
$$

According to the calculation results under the above centralized decision can be obtained: $e_{c}=\varepsilon N / M_{1}$, $\rho_{c}=k N / M_{1} \quad, \quad$ Substitute $\rho_{c}$ and $e_{c}$ into $p=(a+\varepsilon e+b w) / 2 b$ under decentralized decision making, we can get $w_{c}=c$. And then substitute $\rho_{c}, e_{c}$ and $w_{c}$ into equation (11) to get : $\pi_{r c}=b k^{2} N^{2} / M_{1}^{2}-F>0$, so $0 \leq F<b k^{2} N^{2} / M_{1}^{2}$.

\section{NUMERICAL SIMULATION}

This section uses the numerical analysis method to explore the impact of emission reduction cost factors and equity concern parameters on emission reduction level, profit and manufacturer's utility. parameter assignment is as follows: $a=1000, b=50, c=6, \varepsilon=40, \gamma=2, k \in[60,100]$

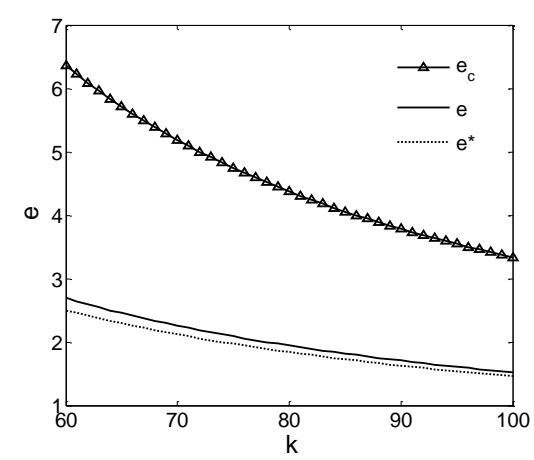

Figure 1 Influence of $k$ on emission reduction level

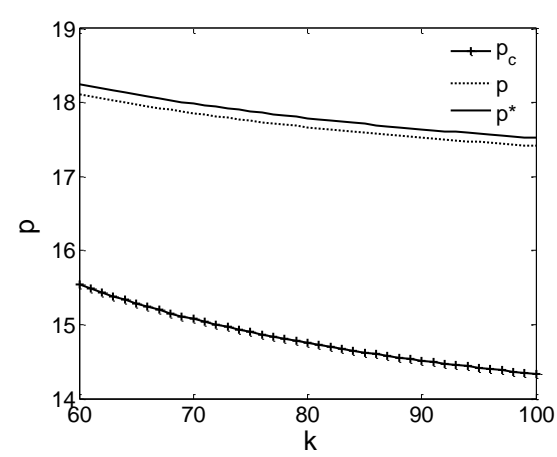

Figure 2 Impact of $k$ on retail prices

As can be seen from figure 1-2, emission reduction level, retail price and emission reduction cost are negatively changing. Therefore, when emission reduction cost increases, manufacturers are reluctant to take emission reduction measures. According to figure 1, the manufacturer's fair concern behaviour will reduce the emission reduction level, and the emission reduction level will be the highest under the centralized decision-making. According to figure 2, the manufacturer's fair concern behaviour is conducive to the increase of wholesale price, 
and the retail price is the lowest under the centralized decision-making.

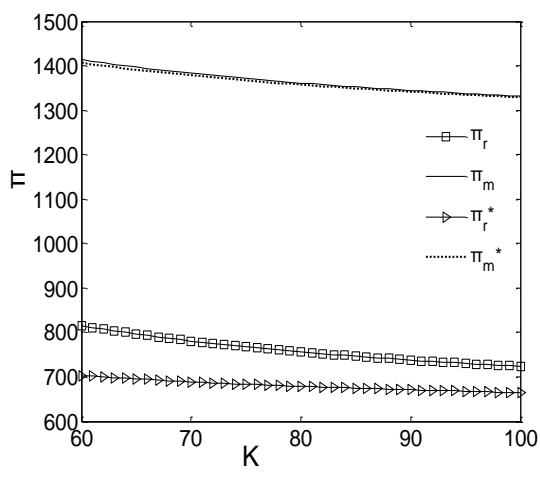

Figure 3 Impact of $k$ on profit

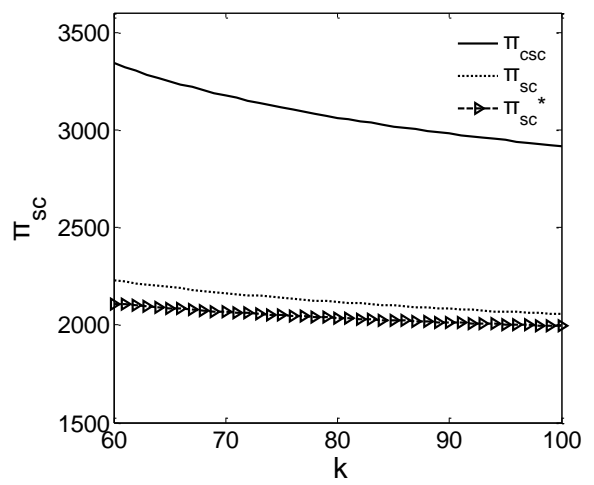

Figure 4 Impact of k on supply chain profit

As can be seen from figures 3 and 4 , the profit and emission reduction cost factors change in a negative direction. According to figure 3, manufacturers' equity concerns are not conducive to the improvement of manufacturers' profits, and manufacturers' profits under the manufacturer-dominated structure are higher than retailers' profits. According to figure 4, equity concern behavior is not conducive to the improvement of supply chain profit, and the supply chain profit is the highest under centralized decision-making.

\section{CONCLUSION}

It can be found by comparing the emission reduction level, sales price and profit level under fair neutrality and fair concern: manufacturers' fair concern behaviour is conducive to reducing emission reduction level, and at the same time is conducive to increasing retail price; manufacturer's profit and retailer's profit decrease with the increase of emission reduction cost factor. Fair concern behaviour is not conducive to the improvement of supply chain and member's profit. Therefore, manufacturer's fair concern behaviour cannot improve the overall utility of supply chain.

\section{ACKNOWLEDGMENT}

This work was supported by Scientific Research Project Funds of Guangdong University Provincial Youth (2018WQNCX277), Scientific research project Funds of Xinhua College of sun yat-sen university (2018YB011).

\section{REFERENCES}

[1] Cui T H, Raju J S, Zhang Z J. Fairness and Channel Coordination, Management Science, 53(8) (2007) 1303 -1314. DOI: https://doi.org/10.1287/mnsc. 1060.0697

[2] DU S F, DU C, LIANG L. Supply chain coordination considering fairness concerns, Journal of Management Sciences in China, 13(11) (2010) 41-48. DOI: http://www.cnki.com.cn/Article/CJFDTotalJCYJ201011006.htm

[3]LI Y, ZHAO D Z. Low Carbonization supply chain coordination with contracts considering fairness preference, Journal of Industrial Engineering and Engineering Management, 29(1)( 2015) 156-161. DOI: 10.3969/j.issn.1004-6062.2015.01.021

[4] LIU Q Y, ZHANG C K. Supply chain two part tariff contract with fairness preference and carbon emissions, Chinese Journal of Management Science, 24(10) (2016)60-68. DOI:

\subsection{1/j.cnki.issn1003-207x.2016.10.007}

[5] SHI S, YAN B. Pricing and coordination decisions in autonomous reduction low-carbon supply chain with fairness concerns, Systems Engineering Theory, Practice, $36(12)(2016) 3079-3091 . D O I:$ 10.12011/1000-6788(2016)12-3079-13

[6] ZHOU Y, HU J S. Decision Analysis of a Dual Channel Green Supply Chain Considering the Fairness Concern, Industrial Engineering and Management, 25(1) (2020) 9-19.DOI:

\subsection{5/j.cnki.1007-5429.2020.01.002}

[7] ZHOU Y J, BAO M J. Co-op advertising and emission reduction cost sharing contracts and coordination in low-carbon supply chain based on fairness concerns, Chinese Journal of Management Science, 25(2) (2017)121-129. DOI:

10.16381/j.cnki.issn1003-207x.2017.02.01 
[8] ZHOU Y J, HU F Y. Study on Optimal Decisions of Retailer-dominated Low-carbon Supply Chain Based on

Fairness

Concern,13(2018)207-214.DOI:

10.3969/j.issn.1000-7695.2018.13.031 